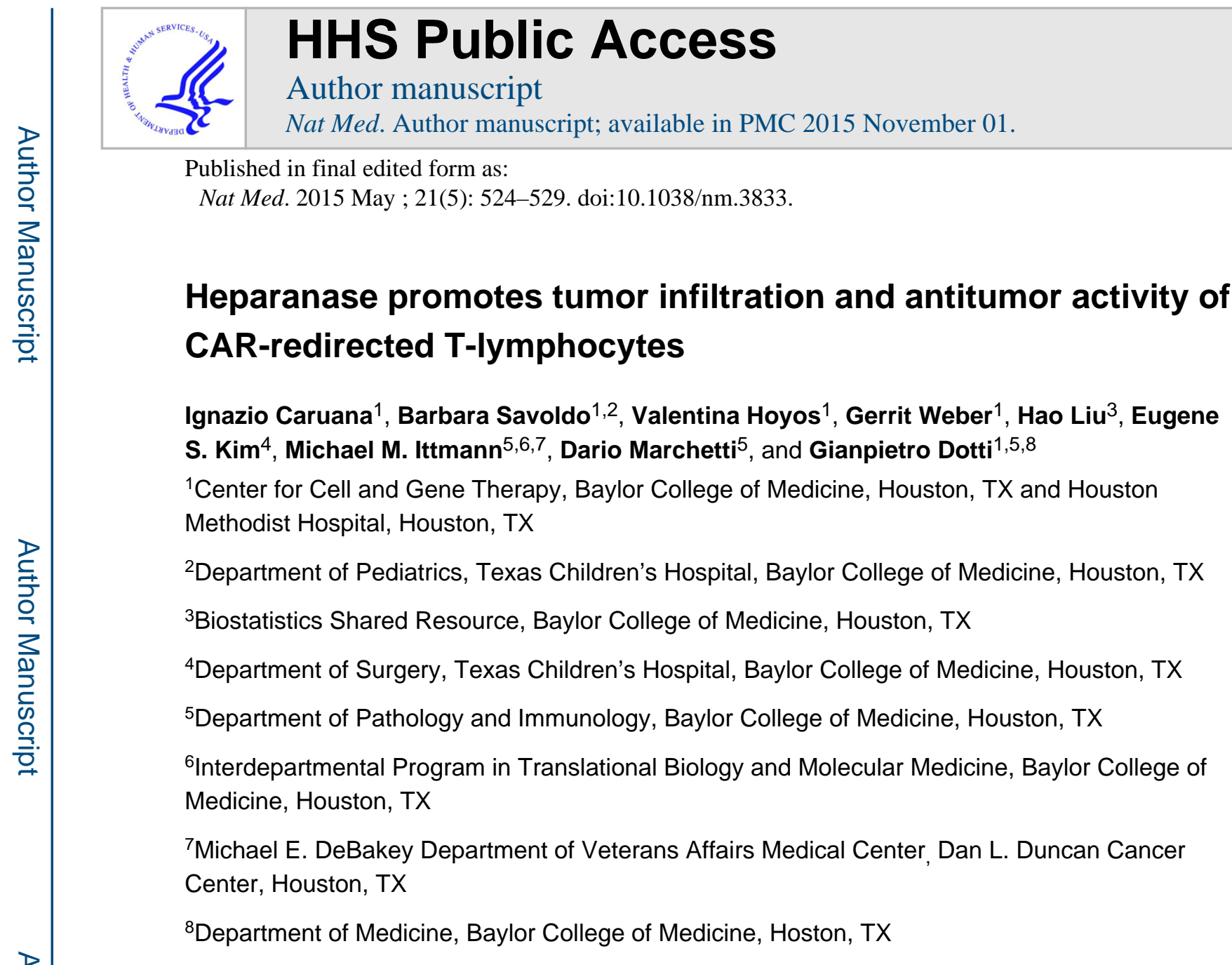

\begin{abstract}
Adoptive transfer of chimeric antigen receptor (CAR)-redirected T lymphocytes (CAR-T cells) has had less striking effects in solid tumors ${ }^{1-3}$ than in lymphoid malignancies ${ }^{4,5}$. Although active tumor-mediated immunosuppression may play a role in limiting efficacy ${ }^{6}$, functional changes in $\mathrm{T}$ lymphocytes following their ex vivo manipulation may also account for cultured CAR-T cells' reduced ability to penetrate stroma-rich solid tumors. We therefore studied the capacity of human in vitro-cultured CAR-T cells to degrade components of the extracellular matrix (ECM). In contrast to freshly isolated $\mathrm{T}$ lymphocytes, we found that in vitro-cultured $\mathrm{T}$ lymphocytes lack expression of the enzyme heparanase (HPSE) that degrades heparan sulphate proteoglycans, which are main components of ECM. We found that HPSE mRNA is down regulated in in vitroexpanded T cells, which may be a consequence of p53 binding to the HPSE gene promoter. We therefore engineered CAR-T cells to express HPSE and showed improved capacity to degrade
\end{abstract}

Users may view, print, copy, and download text and data-mine the content in such documents, for the purposes of academic research, subject always to the full Conditions of use:http://www.nature.com/authors/editorial_policies/license.html\#terms

Address Correspondences to: Gianpietro Dotti, Center for Cell and Gene Therapy, Baylor College of Medicine, 6621 Fannin St. MC 3-3320, Houston, TX 77030, Phone: (832) 824-6891, Fax: (832) 825-4732, gdotti@bcm.edu.

Authorship Contribution: G.D., I.C., and B.S. designed experiments; I.C., V.H., G.W., and E.S. performed the experiments; I.C., B.S. and G.D. analyzed the data; I.C. and G.D. wrote the manuscript; H.L. performed the statistical analysis; M.M.I performed the pathology; D.M. provided his expertize in the heparanase field and provided crucial reagents; all the authors reviewed and approved the final version of the manuscript.

Disclosure conflict-of-interest disclosure: G.D. and B.S. have ownership interest (including patents) in the field of T-cell and genemodified T-cell therapy for cancer and have a collaborative research agreement with Celgene and Bluebird bio. 
ECM, which promoted tumor T-cell infiltration and antitumor activity. Employing this strategy may enhance the activity of CAR-T cells in individuals with stroma-rich solid tumors.

CAR-T cells in vitro-cultured consist mostly of memory and effector lymphocytes characterized by potent cytotoxic function ${ }^{1,4,7}$. However to exploit their effector function CAR-T cells must traffic to and accumulate in tumor sites. These properties involve a complex sequence of events that, beginning with the adhesion of T cells to endothelial cells followed by chemokine-chemokine receptor interactions, modulates their extravasation into antigen-rich tissues ${ }^{8-10}$. During this process, T lymphocytes actively degrade the main components of the subendothelial basement membrane and the extracellular matrix (ECM), including the heparan sulphate proteoglycans (HSPGs) ${ }^{11}$. ECM is an integral component of the stroma, therefore $\mathrm{T}$ cells attacking stroma-rich solid tumors must be able to degrade HSPGs in order to access tumor cells and exert antitumor effects.

The release of specific enzymes by $\mathrm{T}$ cells is fundamental to the degradation of ECM. One of these enzymes is heparanase (HPSE), which is the only known mammalian $\beta$-Dendoglycosidase capable of cleaving the heparan sulphate chains of HSPGs ${ }^{9}, 12-14$. HPSE is first synthesized as a latent precursor protein of $\sim 65 \mathrm{kDa}$ and then cleaved into two subunits of $\sim 8$ and $\sim 50 \mathrm{kDa}$ that heterodimerize to form the enzymatically active protein ${ }^{13}$. HPSE is produced in large amounts by activated $\mathrm{CD} 4^{+} \mathrm{T}$ lymphocytes, neutrophils, monocytes and $\mathrm{B}$ lymphocytes ${ }^{15-17}$. However, the exact contribution of HPSE in mediating the tumor infiltration of in vitro-cultured, tumor-specific T cells remains unexplored.

We first assessed whether ex vivo expanded human $\mathrm{T}$ cells are defective in their capacity to degrade ECM. Using a Matrigel ${ }^{\mathrm{TM}}$-based cell invasion assay, we compared the invasion capacity of freshly isolated resting T cells (FI-T), briefly activated T cells (BA-T) (24 hour activation with OKT3 and anti-CD28 Abs) and long-term ex vivo expanded T cells (LTE-T) (activation with OKT3 and anti-CD28 Abs and ex vivo culture for 12-14 days). Consistent with previously reported data in rodents ${ }^{12}$, BA-T showed superior invasion of ECM compared to FI-T ( $34 \% \pm 8 \%$ vs. $23 \% \pm 8 \%$, respectively; $\mathrm{p}=0.05)$. Conversely, LTE-T had significantly reduced ability to degrade ECM $(8 \% \pm 6 \%)$ compared to both BA-T $(\mathrm{p}=0.01)$ and FI-T ( $\mathrm{p}=0.022$ ) (Fig. 1a). To dissect the mechanisms responsible for this observation we evaluated the expression and function of HPSE in each cell population. In accordance with the cell invasion assay, both $\mathrm{CD}^{+}$and $\mathrm{CD} 8^{+} \mathrm{T}$ cells from $\mathrm{FI}-\mathrm{T}$ and $\mathrm{BA}-\mathrm{T}$ retained the active form of HPSE ( $50 \mathrm{KDa}$ ), while the enzyme was lost in LTE-T by day 2 of culture (Fig. 1b,c). The loss of HPSE expression was not determined by the culture media or cytokines used for T-cell growth, since we observed similar results using either human AB serum or fetal bovine serum, and either IL-2, IL-7 or IL-15 as T-cell growth factors (Supplementary Fig. 1). We also found that the down regulation of HPSE expression in response to stimulation with $\mathrm{OKT} 3$ and anti-CD28 Abs and cytokines is observed in naive $\left(\mathrm{CD}_{45 \mathrm{RA}^{+}}\right)$, central-memory $\left(\mathrm{CD} 45 \mathrm{RO}^{+} \mathrm{CD}^{2} \mathrm{~L}^{+}\right)$and effector-memory $\left(\mathrm{CD} 45 \mathrm{RO}^{+} \mathrm{CD}^{2} 2 \mathrm{~L}^{-}\right)$cells isolated from the peripheral blood suggesting that this is a general phenomenon and non T-cell subset specific (Supplementary Fig. 2). The absence of HPSE protein in LTE-T was associated with the down-regulation of the HPSE mRNA. As shown in Fig. 1d, HPSE mRNA decreased immediately after activation in both $\mathrm{CD}^{+}$and $\mathrm{CD}^{+} \mathrm{T}^{-}$ 
cells compared to $\mathrm{CD} 14^{+}$monocytes ( $\mathrm{p}<0.005$ and $\mathrm{p}<0.031$, respectively) and remained low over the following 14 days of culture. Re-stimulation of LTE-T with OKT3 and anti-CD28 Abs on day 14 of culture did not induce re-expression of either the HPSE mRNA or protein (Fig. 1b,d). The lack of cellular HPSE in LTE-T was also confirmed by the absence of enzymatic activity in the culture supernatant. As shown in Fig. 1e, HPSE enzymatic activity was detected in supernatants collected within the first 72 hours after activation of FI-T. This detection can be attributed to enzyme accumulation in the culture media. However, the enzymatic activity returned to background levels 72 hours later (from $0.34 \pm 0.2 \mathrm{U} \mathrm{ml}$ and $0.45 \pm 0.27 \mathrm{U} \mathrm{ml}$ to $0.22 \pm 0.06 \mathrm{U} \mathrm{ml}$ for both for $\mathrm{CD}^{+}$and $\mathrm{CD}^{+} \mathrm{T}$ cells (Fig. 1e). This observation is in line with previous studies reporting that preformed HPSE protein is stored in an intracellular compartment and released as an early event in response to T-cell activation ${ }^{18}$. We found that HPSE is also absent in Epstein Barr Virus-specific cytotoxic T cells that are stimulated in vitro by antigen-presenting cells, suggesting that HPSE loss in LTE-T is not caused by a supra-physiological activation of these cells mediated by the OKT3 Ab (Supplementary Fig. 2) ${ }^{19}$. Previous studies showed that $p 53$ mutated with loss of function in tumor cells is associated with over-expression of $\operatorname{HPSE}^{20}$. Since there is an accumulation of the full-length $\mathrm{p} 53$ protein in LTE-T ${ }^{20,21}$, we found that the lack of HPSE mRNA expression in LTE-T may be due to the accumulation of the full-length $\mathrm{p} 53$ protein in LTE-T that binds to the HPSE gene promoter (Fig. 1f-h)(Supplementary Fig. 3). The immediate translational implication of these findings is that $\mathrm{T}$ cells in vitro-engineered and cultured for adoptive immunotherapy lacks HPSE expression when infused into the subjects and are thus impaired in their capacity to degrade components of ECM of the stroma. It is also important to note that the cleavage of HPSG chains by HPSE releases preformed stored chemokines into the stroma ${ }^{22,23}$. Since chemokines also guide the migration of $\mathrm{T}$ cells towards their target cells within the tumor microenvironment, the lack of HPSE may further indirectly compromise the antitumor effects of $\mathrm{T}$ cells by reducing their migration.

We thus hypothesized that engineering LTE-T to express HPSE through retroviral gene transfer would improve their invasion capability. LTE-T transduced with the HPSE(I)GFP retroviral vector expressed GFP (Fig. 2a) and HPSE (Fig. 2b). HPSE expression remained high in transduced LTE-T starved from cytokines in culture for more than 10 days, suggesting a stable expression of the transgene (Fig. 2c). In functional assays, HPSE(I)GFP ${ }^{+}$ LTE-T better degraded ECM $(48 \% \pm 19 \%)$ than did control untransduced LTE-T (29\% \pm $18 \%$; $\mathrm{p}=0.025$ ) (Fig. 2d). The addition of the HPSE-inhibitor Heparin $\mathrm{H}^{24}$, confirmed that the invasion of HPSE(I)GFP ${ }^{+}$LTE-T is HPSE-specificity, as invasion was reduced from $74 \% \pm 14 \%$ in the absence of inhibitor to $29 \% \pm 9 \%(\mathrm{p}<0.01)$ in the presence of Heparin H1 (Fig. 2e). We then assessed whether HPSE expression leading to improved cell invasion could be coupled with antitumor specificity. We used neuroblastoma (NB) as a cancer model, since this tumor has been targeted in a clinical trial with a CAR specific for the NBassociated antigen GD2 with some clinical responses ${ }^{1}$. On day 14 of culture, CAR expression was $71 \% \pm 14 \%$ and $56 \% \pm 6 \%$ in LTE-T transduced with CAR and CAR(I)HPSE vectors, respectively (Fig. 3a). HPSE was detected only in LTE-T transduced with the CAR(I)HPSE vector (Fig. 3b). Both CAR ${ }^{+}$and CAR(I)HPSE ${ }^{+}$LTE-T lysed the $\mathrm{GD} 2{ }^{+}$targets human NB cell line LAN-1 $(71 \% \pm 22 \%$ and $41 \% \pm 16 \%$, respectively, at a 20:1 E:T ratio in a ${ }^{51} \mathrm{Cr}$-release assay)(p=ns), and the human NB cell line CHLA-255 (76\% 
$\pm 7 \%$ and $55 \% \pm 13 \%$, respectively)(p=ns), while both showed negligible activity against the GD2 ${ }^{-}$target Raji ( $<8 \%$ ) (Fig. 3c). Control LTE-T lacking the CAR lysed none of these targets. The cytolytic activity was associated with a preserved Th1 cytokine profile as $\mathrm{CAR}^{+}$ and CAR(I)HPSE ${ }^{+}$LTE-T released similar amounts of IFN- $\gamma(927 \pm 328$ and $527 \pm 320 \mathrm{pg}$ $\mathrm{ml} \times 10^{6}$ cells, respectively; $\left.\mathrm{p}=\mathrm{ns}\right)$ and IL-2 $\left(83 \pm 6\right.$ and $61 \pm 27 \mathrm{pg} \mathrm{ml} \times 10^{6}$ cells, respectively, $\mathrm{p}=\mathrm{ns}$ ) in response to stimulation with the GD2 antigen (Fig. 3d). In sharp contrast to their comparable cytolytic function, only CAR(I)HPSE ${ }^{+}$LTE-T degraded ECM $(66 \% \pm 1 \%)$ compared to $\mathrm{CAR}^{+}$or control LTE-T $(13 \% \pm 9 \%$ and $16 \% \pm 10 \%$, respectively) ( $\mathrm{p}=0.004$ and $\mathrm{p}<0.001$ ) (Fig. 3e). To prove ex vivo that LTE-T co-expressing HPSE and CAR have increased antitumor activity in the presence of ECM, we plated LTE-T and tumor cells in a Matrige ${ }^{\mathrm{TM}}$ cell invasion assay, in which LTE-T must degrade ECM to reach and eliminate the tumor targets. After 3 days of culture, both $\mathrm{CAR}^{+}$and CAR(I)HPSE ${ }^{+}$LTE-T eliminated LAN-1 tumor cells equally well in the absence of ECM (insert) $\left(<3 \% \mathrm{GFP}^{+}\right.$tumor cells) compared to control LTE-T $\left(31 \% \pm 6 \% \mathrm{GFP}^{+}\right.$tumor cells)

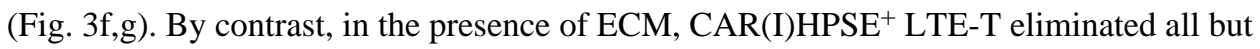
$16 \% \pm 8 \%$ of LAN- 1 cells compared to $37 \% \pm 12 \%$ in the presence of $\mathrm{CAR}^{+} \mathrm{LTE}-\mathrm{T}$ $(\mathrm{p}=0.001)$ (Fig. 3f,g). Control LTE-T did not show antitumor activity in any condition (either insert or ECM) $\left(45 \% \pm 9 \% \mathrm{GFP}^{+}\right.$tumor cells). We obtained identical results with the NB line CHLA-255 (Supplementary Fig. 4). Thus, only LTE-T co-expressing HPSE and CAR show robust antitumor activity in the presence of ECM. The improved antitumor activity of CAR(I)HPSE ${ }^{+}$LTE-T was achieved without causing detectable detrimental effects on $\mathrm{T}$ lymphocytes since these cells expanded in vitro, retained the same phenotype of $\mathrm{CAR}^{+}$LTE-T and did not show increased activation-induced cell death in response to either OKT3 Ab, which binds CD3, or 1A7 anti-idiotype Ab, which causes cross-linking of the GD2-specific CAR ${ }^{1}$ (Supplementary Fig. 5).

To validate our findings in vivo, we first established xenograft models of NB by implanting NSG mice intraperitoneally (i.p.) with either CHLA-255 or LAN-1 NB cell lines in the presence of Matrigel $^{\mathrm{TM}}$ to allow the formation of complex and structured tumors. We utilized the i.p. route to minimize confounding variables related to T-cell homing to the tumor, a known issue in NB xenograft models when the tumor is inoculated subcutaneously ${ }^{25}$. After 10 days, mice received i.p. either control, $\mathrm{CAR}^{+}$or CAR(I)HPSE ${ }^{+}$LTE-T. As shown in Fig. 4a, mice implanted with CHLA-255 tumor cells and treated with CAR(I)HPSE ${ }^{+}$LTE-T had significantly improved survival by day 40 compared to mice treated with control LTE-T $(\mathrm{p}<0.001)$ or $\mathrm{CAR}^{+} \mathrm{LTE}-\mathrm{T}(\mathrm{p}<0.007)$. Among treated mice, we found 6 of 22 infused with $\mathrm{CAR}^{+}$LTE-T, and 18 of 26 infused with CAR(I)HPSE ${ }^{+}$LTE-T macroscopically tumor free at the time we ended the observation by day $40(\mathrm{p}=0.008)$. We obtained similar results in mice engrafted with LAN-1 tumor cells. Mice infused with CAR(I)HPSE ${ }^{+}$LTE-T had significantly improved survival compared to mice treated with control $(\mathrm{p}<0.0001)$ or $\mathrm{CAR}^{+}$ LTE-T at day 50 (p<0.039) (Fig. 4b and Supplementary Fig. 6). In another set of experiments, we euthanized mice on day 12-14 after T-cell infusion to measure tumor T-cell infiltration. Tumors collected from mice infused with CAR(I)HPSE ${ }^{+}$LTE-T had greater Tcell infiltration $(4.6 \% \pm 2.4 \%)$ compared to those treated with control $(0.6 \% \pm 0.5 ; \mathrm{p}=0.029)$ or CAR ${ }^{+}$LTE-T $(0.1 \% \pm 0.1 ; \mathrm{p}=0.043)$ (Fig. 4c). Tumors collected from euthanized mice also showed a significant reduction in weight in recipients infused with CAR(I)HPSE ${ }^{+}$LTE- 
T compared to control ( $0.8 \mathrm{~g} \pm 0.6 \mathrm{~g}$ vs. $3.3 \mathrm{~g} \pm 2.4 \mathrm{~g}$ ) ( $\mathrm{p}=0.039)$ ), and when compared to mice infused with CAR ${ }^{+}$LTE-T $(0.8 \mathrm{~g} \pm 0.6 \mathrm{~g}$ vs. $2.5 \mathrm{~g} \pm 2 \mathrm{~g})$ although this difference was not statistically significant ( $\mathrm{p}=0.093$ ) (Fig. $4 d$ ). Since NB cell lines require Matrigel ${ }^{\mathrm{TM}}$ to form complex and structured tumors when infused i.p., we also implemented a third NB model in which CHLA-255 tumor cells labeled with firefly luciferase were implanted in the kidney of NSG mice without using Matrigel ${ }^{\mathrm{TM}}{ }^{26}$, and CAR ${ }^{+}$LTE-T and CAR(I)HPSE $^{+}$LTE were infused intravenously (i.v). Tumor sections from mice infused i.v. with CAR(I)HPSE ${ }^{+}$ LTE-T showed enhanced infiltration of $\mathrm{CD}^{+} \mathrm{T}$ cells compared to CAR ${ }^{+} \mathrm{LTE}-\mathrm{T}$ (cell numbers $357 \pm 72$ and $173 \pm 32$, respectively; $\mathrm{p}=0.028$ ) (Fig. 4e,f,g). Long-term observation of infused mice also showed improved survival of recipients treated with CAR(I)HPSE ${ }^{+}$ LTE-T by day 50 (p<0.005) (Fig. 4f and Supplementary Fig. 6).

Finally, we extended our observation to $\mathrm{CAR}^{+} \mathrm{LTE}-\mathrm{T}$ targeting the solid tumor-associated antigen chondroitin sulphate proteoglycan 4 (CSPG4) ${ }^{27}$ in an aggressive melanoma model, suggesting that the positive effect of HPSE in CAR-T cells can be exported to other targeted antigens and solid tumors (Supplementary Fig. 7). In contrast, the co-expression of HPSE in LTE-T redirected with a CD19-specific CAR did not appear to play a role in B-lymphoid malignancies, which are generally stroma-poor compared to solid tumors (Supplementary Fig. 8).

Under physiological conditions, HPSE expression by T cells is regulated to avoid tissue damage from T-cell extravasation into non-pathologic tissues ${ }^{16,17,28,29}$. To rule out concerns about non-specific infiltration of normal tissues, such as lung or liver, by HPSEengineered LTE-T, we evaluated in vivo T-cell bio-distribution. For these experiments, we labeled CAR(I)HPSE ${ }^{+}$and $\mathrm{CAR}^{+}$LTE-T with the vector encoding GFP and firefly luciferase and infused them via tail injection. T-cell bio-distribution evaluated by in vivo imaging at different time points after T-cell inoculation and immunohistochemistry at early and late passages did not show differences between the two groups of mice, suggesting no preferential accumulation in lung or liver of HPSE-engineered LTE-T (Supplementary Fig. 9a,b).

In conclusion, HPSE deficiency in in vitro-engineered and cultured tumor-specific LTE-T may limit their antitumor activity in stroma-rich solid tumors. Other enzymes such as metallo-proteases are also involved in modifications of ECM components and may compensate HPSE deficiency ${ }^{30}$. However, we found that some metallo-proteases are also downregulated upon TCR activation and cytokine exposure (Supplementary Fig. 10a,b). We thus suggest that inducing expression of HPSE in LET-T co-expressing a tumor-specific CAR improves their capacity to degrade ECM without compromising their viability, expansion or effector function and promotes increased antitumor activity. The proposed strategy may enhance the antitumor activity of CAR-redirected T cells in subjects with stroma-rich solid tumors. 


\section{On line Methods}

\section{Cell lines}

293T, DU-145 and CHLA-255 cell lines were cultured in IMDM (Gibco, Invitrogen ${ }^{\mathrm{TM}}$, Carlsbad, CA) supplemented with 10\% fetal bovine serum (FBS, HyClone, Thermo Scientific, Pittsburgh, PA) and 2 mM GlutaMax (Invitrogen, Carlsbad, CA). MCF-7, Raji, K562, LAN-1, Daudi and SENMA cells were cultured in RPMI1640 (HyClone) supplemented with $10 \%$ FBS and 2 mM GlutaMax. A549 cells were cultured in DMEM (GIBCO) supplemented with $10 \%$ fetal bovine serum and $2 \mathrm{mM}$ GlutaMax Cells were maintained in a humidified atmosphere containing $5 \% \mathrm{CO}_{2}$ at $37^{\circ} \mathrm{C}$. Tumor cell lines MCF-7, CHLA-255, A549 and DU-145 produced HPSE. All cell lines were routinely tested for mycoplasma and for surface expression of target antigens. Furthermore, all cell lines were authenticated except for CHLA 255 that was established from a neuroblastoma subject and SENMA that was established in our laboratory from a melanoma subject ${ }^{31}$. However, we routinely verified that this line retained the surface expression of the target antigens.

\section{Isolation and culture of primary human T lymphocytes}

Peripheral blood mononuclear cells (PBMC) were isolated from samples obtained from healthy volunteers from the protocol entitled "Humoral and cellular immune responses to tumor-associated antigens (TAA) - healthy blood and skin donors" which is being conducted after approval by the Institutional Review Board of Baylor College of Medicine, or anonymous buffy coats of healthy donors (Gulf Coast Regional Blood Center, Houston, TX) using Lymphoprep ${ }^{\mathrm{TM}}$ density separation (Fresenius Kabi Norge, Oslo, Norway). Monocytes were obtained from PBMC by positive magnetic selection with CD14 microbeads (Miltenyi Biotec, Auburn, $\mathrm{CA}$ ). $\mathrm{CD}^{+}$and $\mathrm{CD}^{+} \mathrm{T}$ cells were also obtained from PBMC by negative magnetic selection (Miltenyi Biotec). In selected experiments, naive $\left(\mathrm{CD} 45 \mathrm{RA}^{+}\right)$, central-memory $\left(\mathrm{CD} 45 \mathrm{RO}^{+} \mathrm{CD} 6 \mathrm{~L}^{+}\right)$and effector-memory $\left(\mathrm{CD} 45 \mathrm{RO}^{+} \mathrm{CD}^{2} 2 \mathrm{~L}^{-}\right) \mathrm{T}$ cells were also separated from PBMC by CD45RA depletion and CD62L positive paramagnetic selection (Miltenyi Biotec). $\mathrm{T}$ lymphocytes were activated with immobilized OKT3 (1 $\mu \mathrm{g} \mathrm{ml})$ and anti-CD28 (\#555725, Becton Dickinson Biosciences, Franklin Lakes, $\mathrm{NJ})(1 \mu \mathrm{g} \mathrm{ml})$ antibodies (Abs) and then expanded in complete medium containing 45\% RPMI1640 and 45\% Click's medium (Irvine Scientific, Santa Ana, CA, USA) supplemented with $10 \%$ FBS and 2 mM GlutaMAX. Cells were fed twice a week with recombinant interleukin-2 (IL-2) (50 U mL) (Chiron Therapeutics, Emeryville, CA). We defined as FI-T: freshly isolated resting $\mathrm{T}$ cells from peripheral blood that comprise naive, effector-memory and central-memory $\mathrm{T}$ cells; BA-T: briefly activated $\mathrm{T}$ cells that result from incubation of FI-T with OKT3 and anti-CD28 Abs for 24 hours; LTE-T: long term ex vivo expanded T cells that result from BA-T cultured ex vivo for 12-14 days and consist mostly of central-memory and effector-memory T cells. At day 14 of culture, LTE-T cells were reactivated with OKT3 and anti-CD28 Abs and cultured for additional 24 hours. For the experiments in which we compared the invasion capacity of FI-T vs. BA-T vs. LTE$\mathrm{T}$ side by side, we obtained 3 separated blood draws at different time points from the donors to make in LTE-T, BA-T and FI-T to be able to run all the samples in parallel in the invasion assay. 


\section{Cell invasion assay}

The capacity of each cell subset to degrade ECM was examined in vitro using the BioCoat ${ }^{\mathrm{TM}}$ Matrigel $^{\mathrm{TM}}$ Invasion assay (Becton Dickinson Biosciences) following the manufacturer's instructions. Five percent FBS was used as a chemoattactant in the low chamber. All experiments were performed in duplicate. Data are expressed as the percentage of invasion through the Matrigel ${ }^{\mathrm{TM}}$ and the membrane relative to the migration through the control membrane ( $8 \mu \mathrm{m}$ polyethylene terephthalate membrane pores). The percentage of invasion was calculated as follows: (mean of cells invading through the Matrigel ${ }^{\mathrm{TM}}$ chamber membrane/mean of cells migrating through the control insert membrane) $\times 100$. In specific experiments, we simultaneously evaluated the invasion and antitumor activity of LTE-T. Briefly, we used the BioCoat ${ }^{\mathrm{TM}}$ Matrigel $^{\mathrm{TM}}$ Invasion assay and plated LAN-1-GFP ${ }^{+}$or CHLA-255-GFP ${ }^{+}$cells $\left(1.4 \times 10^{5}\right)$ in the bottom of a 24-well plate and LTE-T $\left(2.5 \times 10^{5}\right.$ cells) in the upper chamber/insert. Chambers and inserts were removed 24 hours later. After three days of culture cells were then collected from the lower chamber and quantified by flow cytometry to identify tumor cells and T cells, respectively.

\section{Western blot}

Twenty $\mu \mathrm{g}$ of proteins were resolved by SDS-PAGE and transferred to polyvinylidene difluoride membranes (Bio-Rad, Hercules, CA). The Abs and dilutions used in these experiments were as follows: mouse anti-human HPA1-HPSE (1:100 and 1:6500 dilution, clone HP130 and HP3-17, respectively) (InSight Biopharmaceuticals Ltd, Rehovot, Israel) that recognizes both the $65 \mathrm{kDa}$ precursor and the $50 \mathrm{kDa}$ active form of HPSE-1, rabbit antihuman HPA1 polyclonal (1:4000 dilution) (\#CLANT155, Cedarlane, Burlington, NC), mouse anti-human $p 53$ (1:200 dilution, clone DO-1) (Santa Cruz Biotechnology, Santa Cruz, CA) that recognizes the full-length $p 53$ protein, mouse anti-human $\beta$-actin (1:10000 dilution, clone C4) (Santa Cruz Biotechnology) and horseradish peroxidase conjugated secondary Abs (1:5000 dilution, goat anti-mouse \#sc-2005 and goat anti-rabbit \#sc-2004) (Santa Cruz Biotechnology). Blots were then incubated with SuperSignal West Femto Maximum Sensitivity Substrate (Thermo Scientific).

\section{Immunofluorescence}

Cells were fixed with $4 \%$ paraformaldehyde. After permeabilization with $0.1 \%$ Triton $\mathrm{X}-100$, cells were incubated with 5\% goat serum (Cell Signaling Tecnology ${ }^{\circledR}$, Danvers, $\mathrm{MA}$ ) and $1 \%$ BSA to block non-specific binding. Cells were then stained with the primary $\mathrm{Ab}$ against human HPSE1 (HPA1, clone HP130) (InSight Biopharmaceuticals Ltd) (1:100 dilution at $25^{\circ} \mathrm{C}$ for 2 hours). Cells were then probed with Alexa Fluor 555 goat anti-mouse secondary antibody (\#44095, 1:500 dilution at $25^{\circ} \mathrm{C}$ for 2 hours) (Cell Signaling technology ${ }^{\circledR}$ ). Fluorescent signals were detected using a fluorescence microscope (Olympus IX70, Leeds Instruments Inc, Irving, TX). DAPI (BioLegend, San Diego, CA) was used as nuclear staining.

\section{RNA isolation and quantitative real-time PCR (qRT-PCR)}

For the qRT-PCR, $100 \mathrm{ng}$ of total RNA was used to prepare cDNA (TaqMan One Step PCR Master Mix Reagents Kit) (Applied Biosystem, Carlsbad, CA). Specific primers and probes 
that were designed, tested, and standardized by Applied Biosystems for HPSE and $p 53$ were used (HPSE: Hs00935036_m1; p53: Hs01034249_m1). The difference in cycle threshold values $(\triangle \mathrm{CT}$ ) of HPSE was normalized to the $\triangle \mathrm{CT}$ of GAPDH (Glyceraldehude-3-phospate dehydrogenase, Hs99999905_m1), and the fold-change in expression was expressed relative to $\mathrm{CD} 14^{+}$monocytes, considered as a positive control while mesemchymal stem cells were used as a negative control. For $p 53$ mRNA quantification the difference in cycle threshold values $(\triangle \mathrm{CT})$ of $p 53$ was normalized to the $\triangle \mathrm{CT}$ of $G A P D H$ (Glyceraldehyde-3-phospate dehydrogenase, Hs99999905_m1), and the fold-change in expression was expressed relative to FI-T.

\section{Enzyme-linked immunosorbent assay (ELISA)}

Cytokine release by LTE-T in response to stimulation with GD2 ${ }^{+}$LAN-1 cells was analyzed using IFN- $\gamma$ and IL-2 specific ELISAs (R\&D Systems, Minneapolis, MN). HPSE activity was measured using a heparan sulfate (HS) degrading enzyme assay kit (Takara Bio Inc, Otsu, Shiga, Japan). HPSE activity was measured in supernatants collected at different time point of culture of T lymphocytes. At day 4 and 14 of culture, T cells were collected, washed and re-suspended in fresh media. As basal level of HPSE release we used nonactivated $\mathrm{T}$ cells rested for $48-72$ hours in media. Supernatant from $\mathrm{CD} 14^{+}$monocytes and tumor cell lines were used as positive control. HPSE activity was determined as the inverse of decrease in absorbance as previously described ${ }^{32,33}$. T-cell and tumor cell supernatants were analysed in triplicate.

\section{Multiplex for metallo-proteases (MMPs)}

Analysis of MMPs was performed using Milliplex Map kit panel 1 and 2 (Millipore) following the manufacturer's instructions. In particular, FI-T cells were activated with OKT3 and anti-CD28 mAbs in presence of IL-2 [50 U ml or $2000 \mathrm{U} \mathrm{ml}$ ] or IL-7 and IL-15 [10 $\mathrm{ng} \mathrm{ml}$ and $5 \mathrm{ng} \mathrm{ml}$, respectively]. T cells were fed twice a week. At day 3, 7 and 10 of culture, supernatants and T cells were collected and $60 \mu \mathrm{g}$ sample were tested per well.

\section{p53 chromatin immunoprecipitation (ChIP) assay}

LTE-T, CD45RA ${ }^{+}$and CD45RO ${ }^{+}$T cells were collected and fixed with formaldehyde (Merck, Darmstadt, Germany) to a final concentration of $1 \%$. Fixation proceeded at room temperature for $10 \mathrm{~min}$ and was stopped by the addition of glycine to a final concentration of $0.125 \mathrm{~mol} \mathrm{l}$. The cells were then washed twice with cold PBS. Pellets were resuspended in $350 \mu \mathrm{l}$ of lysis buffer and protease inhibitors mixture (Active Motif, Carlsbad, CA), washed and resuspended in $350 \mu \mathrm{l}$ of optimized ChIP lysis buffer and protease inhibitors mixture (0.5\% SDS, 10mM EDTA, $0.5 \mathrm{mM}$ EGTA and $50 \mathrm{mM}$ Tris-HCl, $\mathrm{pH}$ 8) and sonicated into chromatin fragments of an average length of $500 \mathrm{bp}$, as determined empirically by agarose gel electrophoresis of fragmented chromatin samples. Chromatin was kept at $-80^{\circ} \mathrm{C}$. The chromatin solution was incubated with a $p 53$-specific antibody that recognizes the full-length human protein (FL393, Santa Cruz) at $4^{\circ} \mathrm{C}$ overnight with rotation. The immunoprecipitation was performed using Chip-IT ${ }^{\circledR}$ Express kit (Active Motif, Carlsbad, CA) following the manufacturer's instructions. Amplifications (37 cycles) were performed using specific primers (Supplementary Figure 11), yielding PCR products $\approx$ 
$200 \mathrm{bp}$ in length (location of primers relatively to the origin of the promoter is indicated in parentheses after each primer pair). PCR products were separated by $1.5 \%$ agarose electrophoresis in Tris-borate-EDTA buffer and stained with ethidium bromide.

\section{Retroviral constructs, transient transfection and transduction of T lymphocytes}

HPSE cDNA (accession number NM-006665) was cloned into the SFG retroviral backbone that also encodes the GFP [SFG.HPSE(I)GFP] (Supplementary Fig. 12). The construct for the GD2-specific CAR containing the CD28, OX40 and $\zeta$ endodomains was previously described (SFG.CAR) ${ }^{34}$. We then generated a bicistronic vector to co-express the HPSE and CAR-GD2 using an IRES [SFG.CAR(I)HPSE] (Supplementary Fig. 12). The retroviral vector encoding the fusion protein GFP-firefly luciferase (GFP.FFLuc) for in vivo imaging of T cells and CD19-specific and CSPG4-specific CARs were previously described ${ }^{35}$. Transient retroviral supernatant was produced as previously described ${ }^{35}$. A specific inhibitor of HPSE, Roneparstat (SST0001) (a chemically modified heparin ${ }^{100} \mathrm{Na}, \mathrm{Ro}-\mathrm{H}$, property of Sigma-tau Research Switzerland S.A.) $(3 \mu \mathrm{g} \mathrm{ml})^{13}, 36,37$, was added to the media during the virus preparation to increase its titer. Activated T lymphocytes were then transduced with retroviral supernatants using retronectin-coated plates (Takara Bio Inc, Shiga, Japan). After removal from the retronectin plates, $\mathrm{T}$-cell lines were maintained in complete $\mathrm{T}$-cell medium in a humidified atmosphere containing $5 \% \mathrm{CO}_{2}$ at $37^{\circ} \mathrm{C}$ in the presence of IL-2 $(50 \mathrm{U} \mathrm{mL})$ for 2 weeks.

\section{Flow cytometry}

We performed flow cytometry analysis using the following Abs: CD45, CD56, CD8, CD4, CD3, CD45RA, CD45RO, CD62L, 7AAD and Annexin V (all from Becton Dickinson, San Jose, CA) and CCR7 (from E\&D) conjugated with FITC, PE, PerCP or APC fluorochromes. Expression of GD2 and CSPG4 antigens on tumor cell lines was assesed with anti-GD2 (clone 14.g2a, BD) and anti-CSPG4 (Clone 1E6.4, Miltenyi-Biotech), respectively. The expression of GD2-specific CAR was detected using a specific anti-idiotype antibody (1A7). Samples were analyzed with a BD FACScalibur system equipped with the filter set for quadruple fluorescence signals and the CellQuest software (BD Biosciences). For each sample we analyzed a minimum of 10,000 events.

\section{Chromium-release assay}

The cytotoxic activity of $\mathrm{T}$ cells was evaluated using a standard 6-hour ${ }^{51} \mathrm{Cr}$-release assay.

\section{Xenogenic mouse models}

We used NSG mouse model to assess the in vivo antitumor effect of control and transduced T cells. All mouse experiments were approved by Institutional Animal Care and Use Committee of Baylor College of Medicine. Eight-10 week old NSG mice (Jackson Lab, Bar Harbor, Maine) were injected i.p. with either CHLA-255 or LAN-1 neuroblastoma cells $\left(\mathrm{GD}^{+}\right)\left(2.5 \times 10^{6}\right)$ or SENMA melanoma cells $\left(\mathrm{CSPG} 4^{+}\right)\left(5 \times 10^{5}\right)$ resuspended in Matrigel (BD Biosciences). These tumor cell lines were labelled with Firefly luciferase. Ten-12 days after neuroblastoma inoculation and 2 days after melanoma inoculation, $\mathrm{T}$ cells were injected i.p. $\left(2 \times 10^{7}\right.$ cells $\times$ mouse $)$. No randomization was used. Investigators were not 
blinded, but mice were matched based on the signal of tumor cells before assignment to control or treatment groups. Mice were euthanized when signs of discomfort were detected by the investigator or as recommended by the veterinarian who monitored the mice three times a week. When valuable, tumor growth was also monitored by bioluminescence. For the in vivo bio-distribution of T cells, $5 \times 10^{6} \mathrm{~T}$ cells xmouse labelled with the GFP.FFluc vector were infused via tail injection. For in vivo imaging, we used the Xenogen-IVIS Imaging System as previously described ${ }^{35}$. In the orthotopic model ${ }^{26}$, an inoculum of $10^{6}$ CHLA-255 luciferase-transduced tumor cells suspended in $0.1 \mathrm{ml}$ of PBS was surgically implanted under the renal capsule of 5-7 week old female mice using a 27-gauge needle. $\mathrm{CAR}^{+}$and CAR(I)HPSE ${ }^{+}$T cells were infused i.v. (1-1.5 X $10^{7} \times$ mouse) 7 days later, and tumor regression was measured by bioluminescence imaging. By day 10 , some mice were sacrificed and tumors collected, fixed and stained with anti-human CD3 mAb (A0452, Dako North America Inc., Carpinteria, CA). Mice were euthanized when signs of discomfort were detected by the investigator or as recommended by the veterinarian who monitored the mice three times a week or when luciferase signal reached $7.5 \times 10^{7} \mathrm{p} \mathrm{sec} \mathrm{cm}^{2}$ sr to investigate animal survival. For the lymphoma model mice were infused i.v. with Daudi cells $\left(2 \times 10^{6}\right.$ cells) labelled with Firefly luciferase. Four days later they were infused i.v. with $10^{7}$ control or $\mathrm{CAR}^{+}$and $\mathrm{CAR}(\mathrm{I}) \mathrm{HPSE}^{+} \mathrm{T}$ cells specific for the CD19 antigen. Tumor growth was measured by bioluminescence imaging. Mice were euthanized when signs of discomfort were detected by the investigator or as recommended by the veterinarian who monitored the mice twice a week.

\section{Tissue processing and immunohistochemistry}

Tissue samples were fixed, processed and stained according to standard procedures. We performed Hematoxylin and Eosin staining and labeling of human T cells using polyclonal rabbit anti human CD3 mAb (A0452, Dako North America Inc., Carpinteria, CA) and for detection we used Dako LSAB + System-HRP (K0679, Dako). Tumors were scored without knowledge of the treatment used by the pathologist (Michael M. Ittmann) by counting human infiltrating $\mathrm{T}$ lymphocytes in 10 high power fields at the edge of the tumor.

\section{Statistical analyses}

Unless otherwise noted, data are summarized as mean \pm standard deviation (SD). Student $t$ test (two-sided) was used to determine statistically significant differences between samples, with $p$ value $<0.05$ indicating a significant difference. When multiple comparison analyses were required, statistical significance was evaluated by a repeated measures ANOVA followed by a Log-rank (Mantel-Cox) test for multiple comparisons. The mouse survival data were analysed using the Kaplan-Meier survival curve and Fisher's exact test was used to measure statistically significant differences. No valuable samples were excluded from the analyses. Animals were excluded only in the event of their death after tumor implant but before T-cell infusion. Neither randomization nor blinding was done during the in vivo study. However, mice were matched based on the tumor signal for control and treatment groups before infusion of control or gene modified T cells. To compare the growth of tumors over time, bioluminescence signal intensity was log transformed and then compared using a two-sample t-test. In contrast, the analysis of the pathologist Michael M. Ittmann, aimed at quantifying tumor infiltration by human T cells, was performed in a blind fashion. 
We estimated the sample size considering the variation and mean of the samples. We tried to reach a conclusion using as small a sample size as possible. We estimated the sample size to detect a difference in means of 2 standard deviation at the 0.05 level of significance with a $80 \%$ power. Graph generation and statistical analyses were performed using Prism version 5.0d software (GraphPad, La Jolla, CA).

\section{Supplementary Material}

Refer to Web version on PubMed Central for supplementary material.

\section{Acknowledgments}

The authors would like to thank Israel Vlodavsky and Malcolm Brenner for the critical revision of the manuscript and Catherine Gillespie for the editing. This work was supported in part by US National Institutes of HealthNational Cancer Institute (G.D. R01 CA142636), Department of Defense and Technology and Therapeutic Development Award (G.D. W81XWH-10-10425). Leonid Metelitsa kindly provided the CHLA 255 human neuroblastoma cell line.

\section{Reference List}

1. Pule MA, et al. Virus-specific T cells engineered to coexpress tumor-specific receptors: persistence and antitumor activity in individuals with neuroblastoma. Nat Med. 2008; 14:1264-1270. [PubMed: 18978797]

2. Kershaw $\mathrm{MH}$, et al. A phase I study on adoptive immunotherapy using gene-modified $\mathrm{T}$ cells for ovarian cancer. Clin Cancer Res. 2006; 12:6106-6115. [PubMed: 17062687]

3. Bawley VS, et al. T Cells Redirected Against HER2 for Adoptive Immunotherapy for HER2positive Osteosarcoma. Molecular Therapy. 2012; 2010(Supplement 1)(20):S206. Ref Type: Abstract.

4. Kalos M, et al. T cells with chimeric antigen receptors have potent antitumor effects and can establish memory in patients with advanced leukemia. Sci Transl Med. 2011; 3:95ra73.

5. Brentjens RJ, et al. CD19-targeted T cells rapidly induce molecular remissions in adults with chemotherapy-refractory acute lymphoblastic leukemia. Sci Transl Med. 2013; 5:177ra38.

6. Zou W. Immunosuppressive networks in the tumour environment and their therapeutic relevance. Nat Rev Cancer. 2005; 5:263-274. [PubMed: 15776005]

7. Savoldo B, et al. CD28 costimulation improves expansion and persistence of chimeric antigen receptor-modified T cells in lymphoma patients. J Clin Invest. 2011; 121:1822-1826. [PubMed: 21540550]

8. Muller WA. Leukocyte-endothelial-cell interactions in leukocyte transmigration and the inflammatory response. Trends Immunol. 2003; 24:327-334. [PubMed: 12810109]

9. Parish CR. The role of heparan sulphate in inflammation. Nat Rev Immunol. 2006; 6:633-643. [PubMed: 16917509]

10. Yadav R, Larbi KY, Young RE, Nourshargh S. Migration of leukocytes through the vessel wall and beyond. Thromb Haemost. 2003; 90:598-606. [PubMed: 14515179]

11. Bernfield M, et al. Functions of cell surface heparan sulfate proteoglycans. Annu Rev Biochem. 1999; 68:729-777. [PubMed: 10872465]

12. de Mestre AM, Staykova MA, Hornby JR, Willenborg DO, Hulett MD. Expression of the heparan sulfate-degrading enzyme heparanase is induced in infiltrating CD4+ T cells in experimental autoimmune encephalomyelitis and regulated at the level of transcription by early growth response gene 1. J Leukoc Biol. 2007; 82:1289-1300. [PubMed: 17656651]

13. Vlodavsky I, Ilan N, Naggi A, Casu B. Heparanase: structure, biological functions, and inhibition by heparin-derived mimetics of heparan sulfate. Curr Pharm Des. 2007; 13:2057-2073. [PubMed: 17627539] 
14. Yurchenco PD, Schittny JC. Molecular architecture of basement membranes. FASEB J. 1990; 4:1577-1590. [PubMed: 2180767]

15. Fridman R, et al. Soluble antigen induces T lymphocytes to secrete an endoglycosidase that degrades the heparan sulfate moiety of subendothelial extracellular matrix. J Cell Physiol. 1987; 130:85-92. [PubMed: 2433294]

16. Naparstek Y, Cohen IR, Fuks Z, Vlodavsky I. Activated T lymphocytes produce a matrixdegrading heparan sulphate endoglycosidase. Nature. 1984; 310:241-244. [PubMed: 6205275]

17. Vlodavsky I, et al. Expression of heparanase by platelets and circulating cells of the immune system: possible involvement in diapedesis and extravasation. Invasion Metastasis. 1992; 12:112127. [PubMed: 1399400]

18. Bartlett MR, Underwood PA, Parish CR. Comparative analysis of the ability of leucocytes, endothelial cells and platelets to degrade the subendothelial basement membrane: evidence for cytokine dependence and detection of a novel sulfatase. Immunol Cell Biol. 1995; 73:113-124. [PubMed: 7797231]

19. Smith CA, et al. Production of genetically modified Epstein-Barr virus-specific cytotoxic T cells for adoptive transfer to patients at high risk of EBV-associated lymphoproliferative disease. $\mathrm{J}$ Hematother. 1995; 4:73-79. [PubMed: 7633844]

20. Baraz L, Haupt Y, Elkin M, Peretz T, Vlodavsky I. Tumor suppressor p53 regulates heparanase gene expression. Oncogene. 2006; 25:3939-3947. [PubMed: 16474844]

21. Mondal AM, et al. p53 isoforms regulate aging- and tumor-associated replicative senescence in T lymphocytes. J Clin Invest. 2013; 123:5247-5257. [PubMed: 24231352]

22. Gallagher JT. Heparan sulfate: growth control with a restricted sequence menu. J Clin Invest. 2001; 108:357-361. [PubMed: 11489926]

23. Iozzo RV. Matrix proteoglycans: from molecular design to cellular function. Annu Rev Biochem. 1998; 67:609-652. [PubMed: 9759499]

24. Nakajima M, Irimura T, Di FN, Nicolson GL. Metastatic melanoma cell heparanase. Characterization of heparan sulfate degradation fragments produced by B16 melanoma endoglucuronidase. J Biol Chem. 1984; 259:2283-2290. [PubMed: 6698965]

25. Craddock JA, et al. Enhanced tumor trafficking of GD2 chimeric antigen receptor T cells by expression of the chemokine receptor CCR2b. J Immunother. 2010; 33:780-788. [PubMed: 20842059]

26. Patterson DM, Shohet JM, Kim ES. Preclinical models of pediatric solid tumors (neuroblastoma) and their use in drug discovery. Curr Protoc Pharmacol. 2011; Chapter 14(Unit)

27. Geldres $\mathrm{C}$, et al. T lymphocytes redirected against the chondroitin sulfate proteoglycan-4 control the growth of multiple solid tumors both in vitro and in vivo. Clin Cancer Res. 2014; 20:962-971. [PubMed: 24334762]

28. Arvatz G, Barash U, Nativ O, Ilan N, Vlodavsky I. Post-transcriptional regulation of heparanase gene expression by a 3' AU-rich element. FASEB J. 2010; 24:4969-4976. [PubMed: 20798248]

29. Lu WC, Liu YN, Kang BB, Chen JH. Trans-activation of heparanase promoter by ETS transcription factors. Oncogene. 2003; 22:919-923. [PubMed: 12584571]

30. Wilson TJ, Singh RK. Proteases as modulators of tumor-stromal interaction: primary tumors to bone metastases. Biochim Biophys Acta. 2008; 1785:85-95. [PubMed: 18082147]

31. Yvon E, et al. Immunotherapy of metastatic melanoma using genetically engineered GD2-specific T cells. Clin Cancer Res. 2009; 15:5852-5860. [PubMed: 19737958]

32. Roy M, et al. Antisense-mediated suppression of Heparanase gene inhibits melanoma cell invasion. Neoplasia. 2005; 7:253-262. [PubMed: 15799825]

33. Zhang L, Sullivan P, Suyama J, Marchetti D. Epidermal growth factor-induced heparanase nucleolar localization augments DNA topoisomerase I activity in brain metastatic breast cancer. Mol Cancer Res. 2010; 8:278-290. [PubMed: 20164500]

34. Pule MA, et al. A chimeric T cell antigen receptor that augments cytokine release and supports clonal expansion of primary human T cells. Mol Ther. 2005; 12:933-941. [PubMed: 15979412]

35. Vera J, et al. T lymphocytes redirected against the kappa light chain of human immunoglobulin efficiently kill mature B lymphocyte-derived malignant cells. Blood. 2006; 108:3890-3897. [PubMed: 16926291] 
36. Naggi A, et al. Modulation of the heparanase-inhibiting activity of heparin through selective desulfation, graded N-acetylation, and glycol splitting. J Biol Chem. 2005; 280:12103-12113. [PubMed: 15647251]

37. Zhang L, Ngo JA, Wetzel MD, Marchetti D. Heparanase mediates a novel mechanism in lapatinibresistant brain metastatic breast cancer. Neoplasia. 2015; 17:101-113. [PubMed: 25622903] 


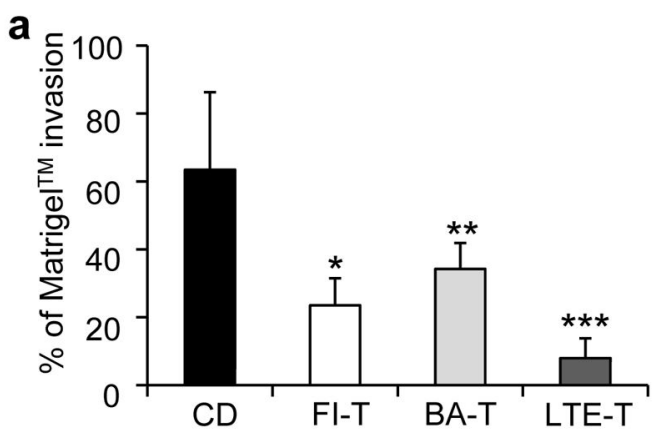

b

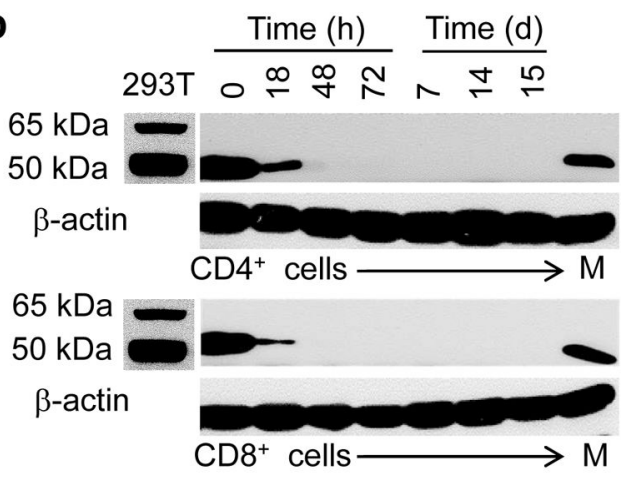

C

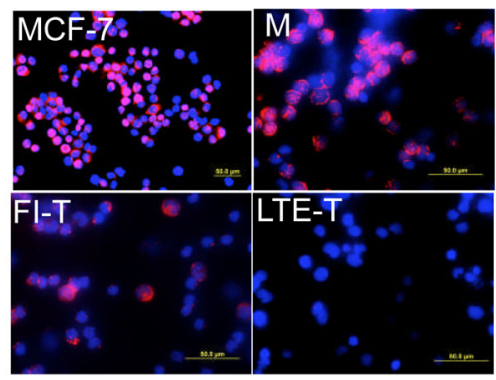

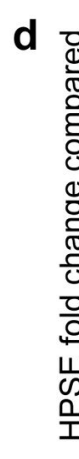

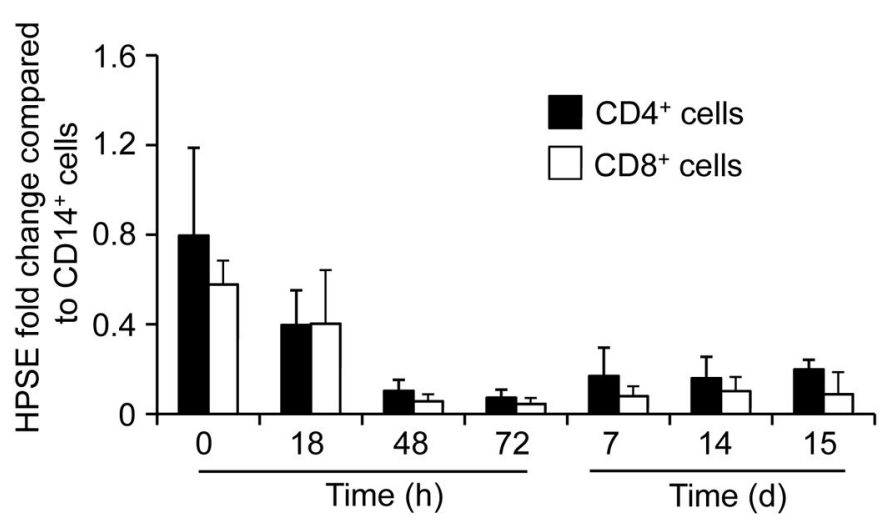

e
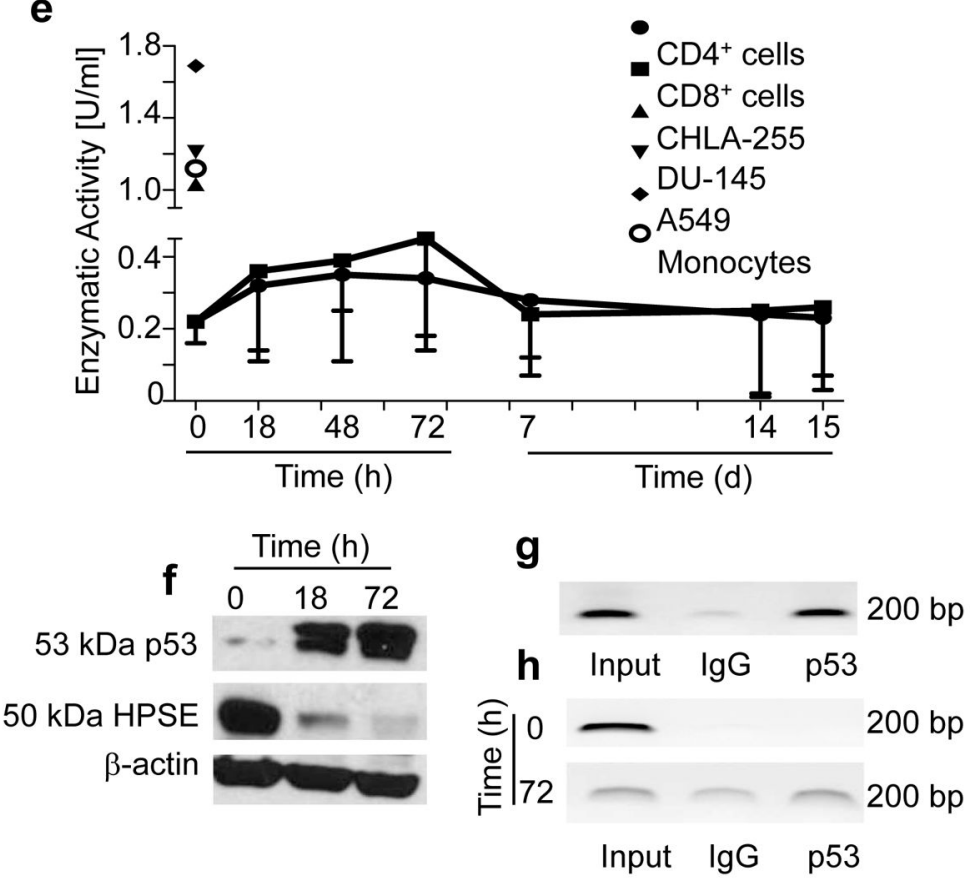

Figure 1. LTE-T show reduced invasion of ECM and loss of the enzyme HPSE

(a) ECM invasion assay of CD14+ monocytes (M), freshly isolated T lymphocytes (FI-T), briefly activated $\mathrm{T}$ cells (BA-T) and long-term ex vivo expanded T cells (LTE-T).

Monocytes freshly isolated from peripheral blood showed the highest capacity to degrade ECM $(63 \% \pm 23 \%)$. BA-T showed superior invasion of ECM compared to FI-T $(* \mathrm{p}=0.05)$.

Conversely, LTE-T had significantly reduced ability to degrade ECM compared to both BA$\mathrm{T}(* * \mathrm{p}=0.01)$ and FI-T $(* * * \mathrm{p}=0.022)$. Data summarize means $\pm \mathrm{SD}$ of 5 donors. We compared all four cell subsets for each donor. (b) Western blot showing the expression of HPSE in $\mathrm{M}, \mathrm{CD}^{+}$and $\mathrm{CD}^{+} \mathrm{T}$ cells at different time points of culture. Data are representative of 4 donors. Positive controls are HPSE transfected 293T cells. (c) Immunofluorescence staining for HPSE in MCF-7 cells, M, FI-T and LTE-T. Nuclei are stained with DAPI and shown in blue, while HPSE is stained with red-fluorescent dye (Alexa Fluor 555). Magnification is 20× (50 micron scale bar). (d) qRT-PCR of HPSE in $\mathrm{CD}^{+}$and $\mathrm{CD}^{+}{ }^{+} \mathrm{T}$ cells at different time points of culture. Data summarize means \pm SD of 4 donors. (e) HPSE enzymatic activity assessed in supernatants collected from $\mathrm{CD}^{+}$and 
$\mathrm{CD} 8^{+} \mathrm{T}$ cells at different time points of culture. Monocytes and tumor cell lines CHLA-255, A549 and DU-145 are positive controls. Data summarize means \pm SD of 4 donors. In (b), (d), (e) the condition "day 15" indicates HPSE expression in LTE-T cultured for 14 days, and re-stimulated with immobilized OKT3 and anti-CD28 Abs for $24 \mathrm{hrs}$ to assess whether TCR re-stimulation can re-induce HPSE expression. (f) Western blot showing the expression of HPSE and full-length $\mathrm{p} 53$ protein in $\mathrm{T}$ cells before $(\mathrm{T}=0)$ and after activation with immobilized OKT3 and anti-CD28 Abs for 18 and 72 hrs. Shown are results from 1 representative of 3 donors. (g) p53 ChIP in LTE-T cultured for 14 days, and (h) in $\mathrm{CD}^{2} 5 \mathrm{RA}^{+}$cells before $(\mathrm{T}=0)$ and after stimulation with OKT3 and anti-CD28 mAbs $(\mathrm{T}=72 \mathrm{~h})$. Input is DNA sonicated but not immunoprecipitated; IgG and $p 53$ are DNA immunoprecipitated by the isotype and $p 53$-specific $\mathrm{Ab}$ that recognizes the full-length protein. Relative quantification was performed comparing the intensities of PCR bands of IgG and $p 53$ to input PCR band. For this representative sample relative quantifications are: IgG $20 \%$ and $p 5390 \%$ for LTE-T (g); IgG $2 \%$ and $p 534 \%$ at $\mathrm{T}=0$ and $\operatorname{IgG} 53 \%$ and $p 53$ $100 \%$ at $\mathrm{T}=72 \mathrm{~h}$ for CD45RA ${ }^{+}$cells $(\mathbf{h})$. Shown is 1 representative of 3 donors. 


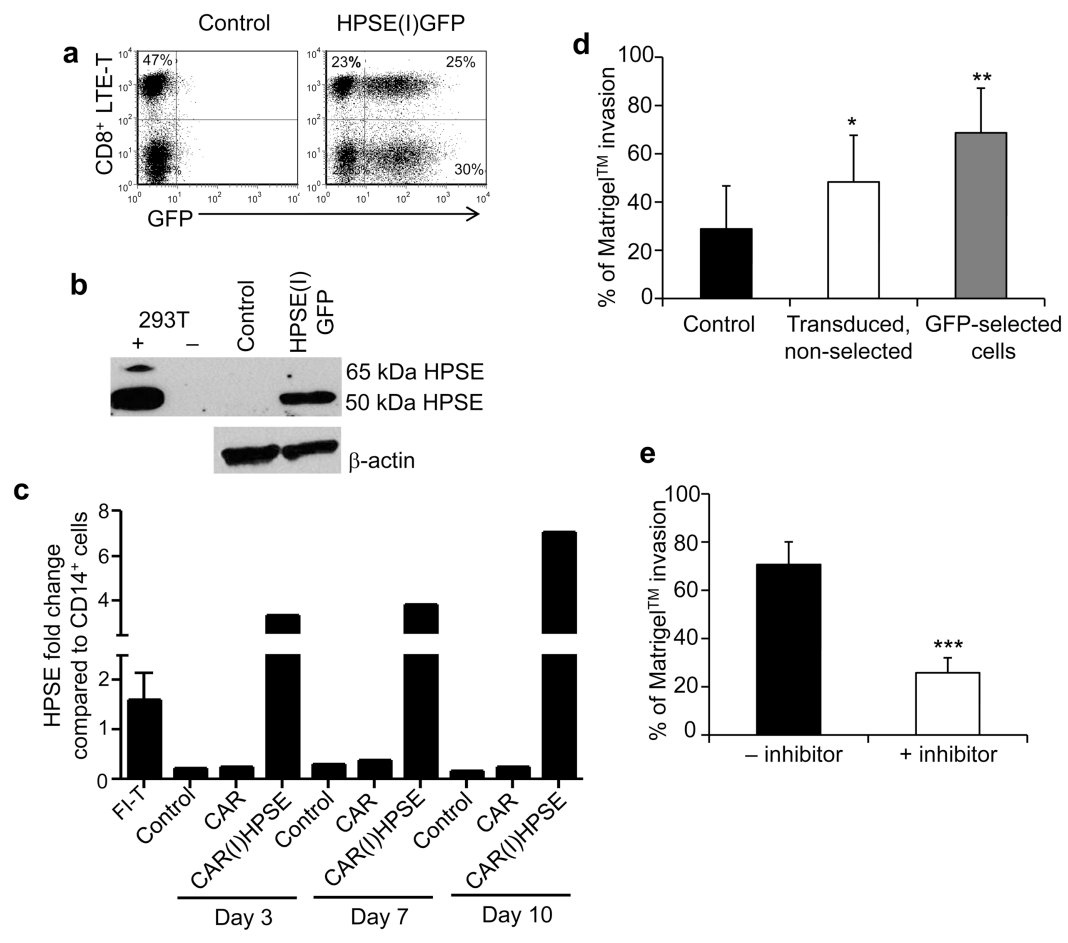

Figure 2. LTE-T modified to express HPSE show enhanced degradation of ECM

We transduced LTE-T with a retroviral vector encoding HPSE and GFP [HPSE(I)GFP]. (a) GFP expression by both CD4+ and CD8 ${ }^{+}$LTE-T at day $12-14$ of culture. (b) Western blot showing the expression of HPSE in control and transduced LTE-T at day $12-14$ of culture. (c) qRT-PCR for HPSE in control and HPSE(I)GFP ${ }^{+}$LTE-T starved in culture from IL-2 for 3, 7 and 10 days. Data are representative of 2 donors. (d) ECM invasion assay of control and HPSE(I)GFP ${ }^{+}$LTE-T, with or without selection based on GFP expression. Data summarize mean \pm SD of 9 donors, $* \mathrm{p}=0.025 ; * * \mathrm{p}<0.001$. (e) ECM invasion assay of HPSE-transduced LTE-T in the presence or in the absence of the inhibitor, heparin H1. Data summarize mean $\pm \mathrm{SD}$ of 4 donors, $* * * \mathrm{p}<0.01$. 

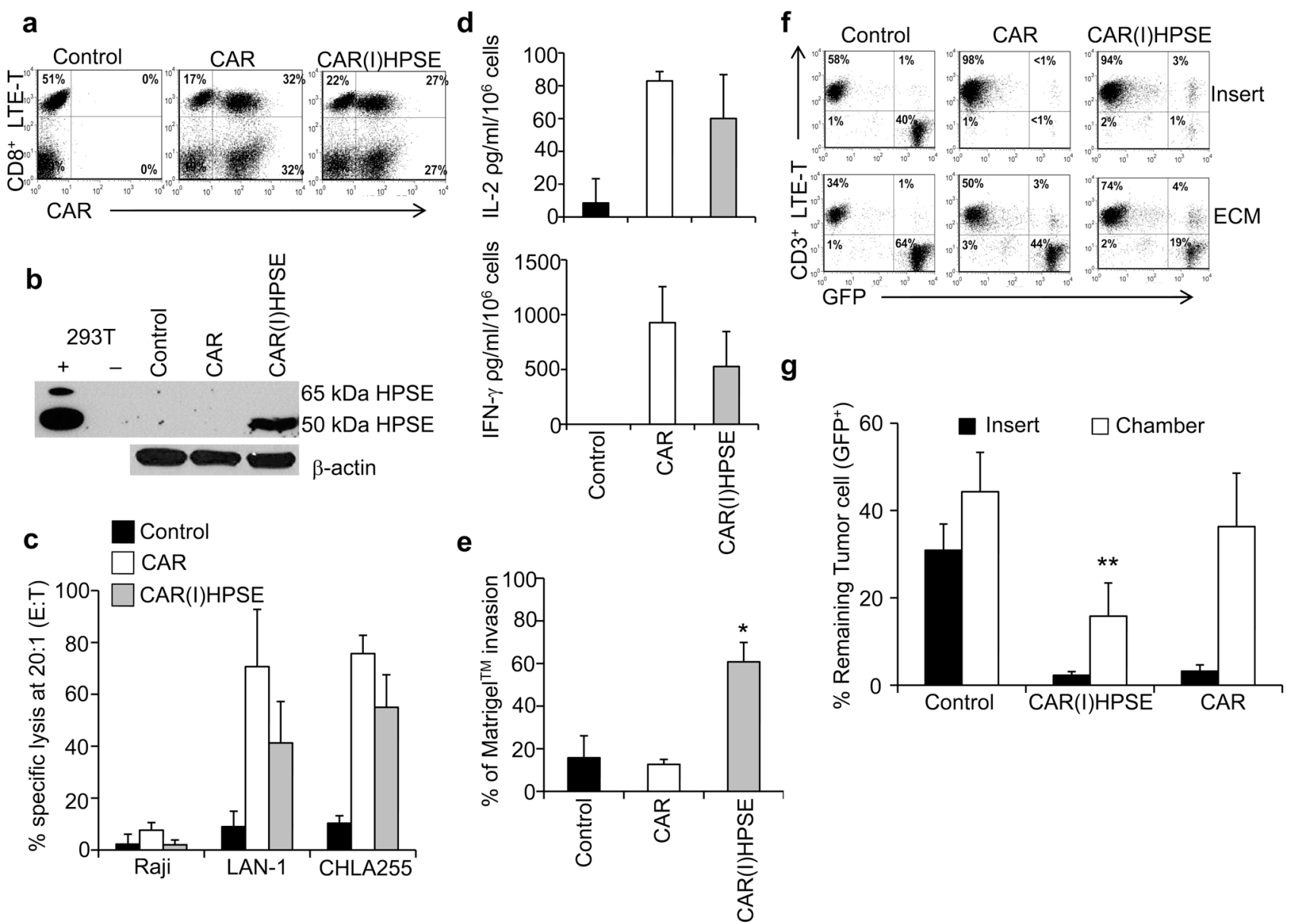

Figure 3. LTE-T co-expressing HPSE and GD2-specific CAR retain GD2 specificity and have enhanced capacity to degrade ECM

We transduced LTE-T with retroviral vectors encoding either the GD2-specific CAR alone (CAR) or both the GD2-specific CAR and HPSE [CAR(I)HPSE]. (a) Flow cytometry analysis to detect CAR expression by control and transduced LTE-T. (b) Western blot to detect HPSE in control and transduced LTE-T. Data are representative of 5 donors. (c) Cytotoxic activity of control, $\mathrm{CAR}^{+}$and $\mathrm{CAR}^{+} \mathrm{HPSE}^{+}$LTE-T assessed by ${ }^{51} \mathrm{Cr}$-release assay at a 20:1 effector:target ratio. We used LAN-1 and CHLA-255 (GD2 ${ }^{+}$), and Raji $\left(\mathrm{GD}^{-}\right)$as target cells. (d) Transduced LTE-T release both IL-2 and IFN- $\gamma$ in response to $\mathrm{GD}^{+}$tumor cells. (e) Invasion of ECM by control, $\mathrm{CAR}^{+}$and $\mathrm{CAR}^{+} \mathrm{HPSE}^{+} \mathrm{LTE}^{-\mathrm{T}}$. Data in (c-e) summarize mean \pm SD from 5 donors, $* \mathrm{p}=0.004$. (f,g) We plated control and transduced LTE-T in the upper part of either ECM assay or insert assay, and LAN-1-GFP+ cells in the lower chamber. After day 3 of culture, we collected cells in the lower chamber to quantify $\mathrm{CD}^{+} \mathrm{T}$ cells and $\mathrm{GFP}^{+}$tumor cells by flow cytometry. (f) illustrates representative dot plots, while (g) summarizes mean \pm SD of 5 donors, $* * p=0.009$. In all cases we analyzed LTE-T by day $12-14$ of culture. 

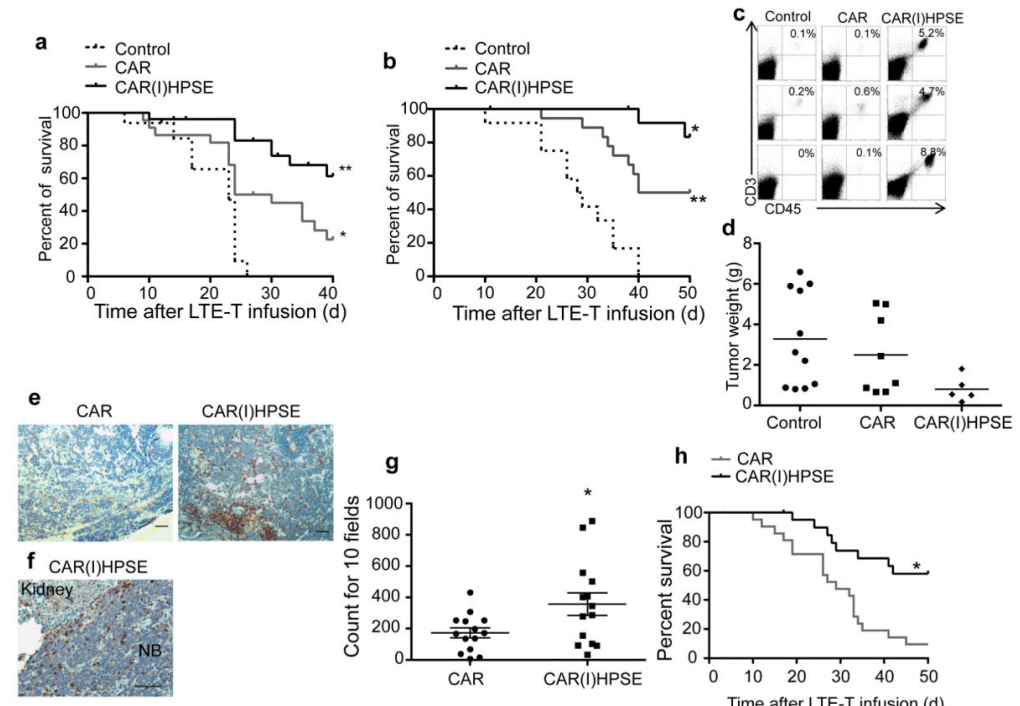

CAR(I)HPSE

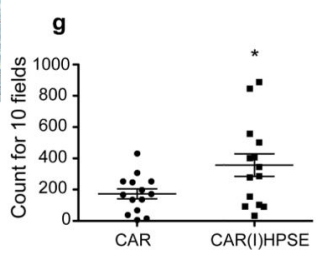

h - CAR

$$
\begin{aligned}
& \text { - CAR } \\
& \text { - CAR(I)HPSE }
\end{aligned}
$$

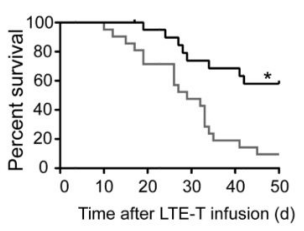

Figure 4. CAR-GD2 ${ }^{+}$LTE-T co-expressing HPSE show enhanced tumor infiltration and improve overall survival in xenograft tumor models

(a) Kaplan-Meier analysis of mice engrafted i.p. with the tumor cell line CHLA-255 and

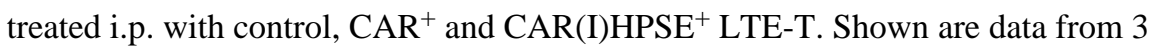
independent experiments using LTE-T generated from 3 donors; control $n=16$, CAR $n=22$, CAR(I)HPSE $\mathrm{n}=26$ mice; $* \mathrm{p}<0.007, * * \mathrm{p}<0.0001$. (b) Kaplan-Meier analysis of mice engrafted i.p. with the tumor cell line LAN-1 and treated i.p. with control, $\mathrm{CAR}^{+}$and CAR(I)HPSE ${ }^{+}$LTE-T. For these experiments, we generated LTE-T from 2 donors; control $\mathrm{n}=12$, CAR $\mathrm{n}=18, \mathrm{CAR}(\mathrm{I}) \mathrm{HPSE} \mathrm{n}=14$ mice; $* \mathrm{p}=0.039$, ** $\mathrm{p}<0.0001$. (c) Flow cytometry analysis of $\mathrm{CD}^{+} \mathrm{T}$ cells detected within the tumor samples. Dot plots are representative of 3 mice per group from mice infused with LTE-T generated from the same donor. (d) Weight of the tumors collected from euthanized mice engrafted with LAN-1 tumor. (e,f) Immunohistochemistry showing $\mathrm{CD}^{+} \mathrm{T}$-cell infiltration in tumors implanted in the kidney of mice infused with either $\mathrm{CAR}^{+}$or CAR-GD2 ${ }^{+} \mathrm{HPSE}^{+} \mathrm{LTE}^{-T}$. $100 \times$ magnification $(\mathbf{e})$ and $200 \times$ magnification (f) (100 micron scale bar). (g) The graph shows the numbers of infiltrating $\mathrm{CD}^{+} \mathrm{T}$ cells per 10 high power fields in tumors collected from mice treated with either $\mathrm{CAR}^{+}$or CAR(I)HPSE ${ }^{+} \mathrm{LTE}^{\mathrm{T}}$, ${ }^{*} \mathrm{p}=0.028$. (h) Kaplan-Meier analysis of tumorbearing mice in the kidney infused i.v. with either $\mathrm{CAR}^{+}$or CAR(I)HPSE ${ }^{+}$LTE-T. For these experiments, we generated LTE-T from 2 donors; CAR $n=21$, CAR(I)HPSE $n=21$ mice; $* \mathrm{p}=0.0006$. 